\title{
A MAXIMAL OPERATOR AND A COVERING LEMMA ON NON-COMPACT SYMMETRIC SPACES
}

\author{
Alexandru D. IONESCU
}

ABstract. The purpose of this paper is to investigate $L^{p}$ boundedness properties of a maximal operator on non-compact symmetric spaces and prove a related covering lemma.

\section{Introduction}

Let $\mathbb{G}$ be a non-compact connected semisimple Lie group with finite center, $\mathbb{K}$ a maximal compact subgroup and $\mathbb{X}=\mathbb{G} / \mathbb{K}$ a non-compact symmetric space. The group $\mathbb{G}$ acts by left translations on the space $\mathbb{X}$ and induces a $\mathbb{G}$-invariant measure $d z$ on $\mathbb{X}$. One also has a distance function $d: \mathbb{X} \times \mathbb{X} \rightarrow \mathbb{R}_{+}$induced by the Killing form on the Lie algebra $\mathfrak{g}$ of the Lie group $\mathbb{G}$. For each $z \in \mathbb{X}$ and $r>0$ let $B(z, r)=\left\{z^{\prime} \in \mathbb{X}: d\left(z, z^{\prime}\right)<r\right\}$ be the ball centered at $z$ of radius $r$ and let $\mathcal{F}$ be the set of all balls $B(z, r), z \in \mathbb{X}, r>0$. For any locally integrable function $f$ on $\mathbb{X}$ let

$$
\mathcal{M}_{\mathcal{F}} \mathcal{F}(z)=\sup _{z \in B \in \mathcal{F}} \frac{1}{|B|} \int_{B}\left|f\left(z^{\prime}\right)\right| d z^{\prime}
$$

where $|B|$ denotes the measure of the set $B \subset \mathbb{X}$. In this paper we will study the question of $L^{p}$ boundedness of the maximal operator $\mathcal{M}_{\mathcal{F}}$ and prove the following theorem:

Theorem 1a. The maximal operator $\mathcal{M}_{\mathcal{F}}$ is bounded from $L^{p}(\mathbb{X})$ to $L^{p}(\mathbb{X})$ in the sharp range of exponents $p \in(2, \infty]$.

We recall that the centered maximal operator

$$
\mathcal{M} f(z)=\sup _{r>0} \frac{1}{|B(z, r)|} \int_{B(z, r)}\left|f\left(z^{\prime}\right)\right| d z^{\prime},
$$

is bounded from $L^{1}(\mathbb{X})$ to $L^{1, \infty}(\mathbb{X})$ and from $L^{p}(\mathbb{X})$ to $L^{p}(\mathbb{X})$ for all $p>1$ as shown in [7] and [1]. However, unlike in Euclidean spaces, balls on symmetric spaces do not have the doubling property (i.e., $|B(z, 2 r)|$ is not proportional to $|B(z, r)|$ if $r$ is large) thus the two maximal operators $\mathcal{M}_{\mathcal{F}}$ and $\mathcal{M}$ are not comparable.

Received October 26, 1999.

The author was supported by an Alfred P. Sloan graduate fellowship. 
A simple connection between boundedness of maximal operators and covering lemmata is explained in [2]. In our setting we have the following equivalent formulation of Theorem 1a:

Theorem 1b. If a collection of balls $B_{i} \in \mathcal{F}, i \in I$ has the property that $\left|\cup B_{i}\right|<$ $\infty$ then one can select a finite subset $J \subset I$ such that

$$
\begin{aligned}
& \text { (i) } c\left|\cup_{i \in I} B_{i}\right| \leq\left|\cup_{j \in J} B_{j}\right| ; \\
& \text { (ii) }\left\|\left.\left|\sum_{j \in J} \chi_{B_{j}} \| C_{L^{q(\mathbb{X})}} \leq C_{q}\right| \cup_{i \in I} B_{i}\right|^{1 / q}, q \in[1,2) .\right.
\end{aligned}
$$

In the terminology of [2] the family $\mathcal{F}$ of balls on $\mathbb{X}$ has the covering property $V_{q}, 1 \leq q<2$. The inequality (ii) in (1.1) is the natural analog of the requirement that the selected balls are disjoint: if $B_{i}, i \in I$ are standard balls in some Euclidean space, then one can select disjoint balls $B_{j}, j \in J$ that satisfy inequality (i) in (1.1). Notice that the disjointness property of the balls $B_{j}$ is equivalent to

$$
\left\|\sum_{j \in J} \chi_{B_{j}}\right\|_{L^{\infty}} \leq\left\|\chi_{\cup B_{i}}\right\|_{L^{\infty}} .
$$

Since balls on symmetric spaces do not have the basic doubling property the disjointness property of the selected balls has to be replaced by (1.1)(ii).

We will prove in the last section of this paper that the maximal operator $\mathcal{M}_{\mathcal{F}}$ is not bounded from the Lorentz space $L^{2, \alpha}(\mathbb{X})$ to $L^{2, \infty}(\mathbb{X})$ if $\alpha>1$. As a consequence the ranges of $p \in(2, \infty]$ for which $\mathcal{M}_{\mathcal{F}}$ is bounded on $L^{p}$ and $q \in[1,2)$ for which the family $\mathcal{F}$ has the covering property $V_{q}$ are best possible. On the other hand, it is proved by a different method in [4] that the maximal operator $\mathcal{M}_{\mathcal{F}}$ is bounded from $L^{2,1}(\mathbb{X})$ to $L^{2, \infty}(\mathbb{X})$ if, in addition, the group $\mathbb{G}$ has real rank one. The author does not know however whether this endpoint estimate holds in the general case.

This work was originally started in collaboration with Jean-Philippe Anker. I would like to thank to him for a number of most clarifying discussions on the structure of semisimple Lie groups and symmetric spaces of high real rank and for explaining to me some of the related methods. I would also like to thank to Elias M. Stein for pointing out to me the papers [2] and [5] that play an essential role in the proofs.

\section{Preliminaries}

In this section we summarize some of the standard notation related to noncompact semisimple Lie groups and state two propositions that will be needed in the proof of Theorem $1 \mathrm{~b}$ in the next section. We start by rewriting Proposition 1 in [2] in a setting suitable for our purposes. Let $X$ be a manifold with a measure $d \nu$ such that open sets are measurable, $\nu(K)<\infty$ for any compact set $K \subset X$ and $\nu(O)=\sup \nu(K)$ for any measurable set $O$ where the supremum is taken 
over all compact subsets $K \subset O$. Let $F$ be a family of open subsets of $X$ of finite measure and assume that $r, s \in(1, \infty)$ are such that $1 / r+1 / s=1$.

Proposition 2. The following statements are equivalent:

(1) The maximal operator

$$
M_{F} f(x)=\sup _{x \in D \in F} \frac{1}{\nu(D)} \int_{D}|f(y)| d \nu(y),
$$

is bounded from $L^{r}(X, d \nu)$ to $L^{r, \infty}(X, d \nu)$.

(2) Given a finite collection of sets $D_{i} \in F, i \in I, I$ finite, one can select a subset $J \subset I$ such that

$$
\begin{aligned}
& \text { (i) } c \nu\left(\cup_{i \in I} D_{i}\right) \leq \nu\left(\cup_{j \in J} D_{j}\right) ; \\
& \text { (ii) }\left\|\sum_{j \in J} \chi_{D_{j}}\right\|_{L^{s}(X, d \nu)} \leq C \nu\left(\cup_{i \in I} D_{i}\right)^{1 / s} .
\end{aligned}
$$

As in [2], we will say that the family $F$ has the covering property $V_{s}$ if it satisfies part (2) of the proposition. In addition, assuming that the maximal operator $M_{F}$ is bounded from $L^{r}(X, d \nu)$ to $L^{r, \infty}(X, d \nu)$ then the selection algorithm given in [2] guarantees that for all $i \in I$

$$
\left|D_{i} \cap\left(\underset{j \in J}{\cup} D_{j}\right)\right| \geq\left|D_{i}\right| / 2 .
$$

We now turn to the structure of the group $\mathbb{G}$. Most of our notation is standard and can be found, for example, in [3]. Let $\mathfrak{g}$ be the Lie algebra of $\mathbb{G}, \theta$ a Cartan involution of $\mathfrak{g}$ and let $\mathfrak{g}=\mathfrak{k} \oplus \mathfrak{p}$ be the associated Cartan decomposition. Let $\mathfrak{a}$ be a maximal abelian subspace of $\mathfrak{p}, \ell=\operatorname{dim}_{\mathbb{R}} \mathfrak{a}$ the real rank of the group $\mathbb{G}, \Sigma$ the restricted root system of the pair $(\mathfrak{g}, \mathfrak{a})$ and $W$ the associated Weyl group. We fix once and for all a positive Weyl chamber $\mathfrak{a}^{+}$and let $\Sigma^{+}$, respectively $\Sigma_{0}^{+}$, denote the corresponding set of positive, respectively simple positive, roots. For any root $\alpha \in \Sigma$ let $\mathfrak{g}_{\alpha}$ be the root space associated to $\alpha$ and let $\mathfrak{n}$ be the direct sum of positive root spaces $\mathfrak{g}_{\alpha}, \alpha \in \Sigma^{+}$. Let $\overline{\mathfrak{n}}=\theta(\mathfrak{n}), \mathbb{N}=\exp \mathfrak{n}$ and $\overline{\mathbb{N}}=\exp \overline{\mathfrak{n}}$.

The group $\mathbb{G}$ has an Iwasawa decomposition $\mathbb{G}=\mathbb{K}(\exp \mathfrak{a}) \mathbb{N}$ and a Cartan decomposition $\mathbb{G}=\mathbb{K}\left(\exp \overline{\mathfrak{a}^{+}}\right) \mathbb{K}$. For each $g \in \mathbb{G}$ denote by $H(g) \in \mathfrak{a}$ and $g^{+} \in \overline{\mathfrak{a}^{+}}$the middle components of $g$ in these decompositions. It is well known that the functions $g \rightarrow H(g)$, respectively $g \rightarrow g^{+}$, are continuous functions from $\mathbb{G}$ to $\mathfrak{a}$, respectively to $\mathfrak{\mathfrak { a }}^{+}$.

The Iwasawa decomposition $\mathbb{G}=\overline{\mathbb{N}} \mathbb{A} \mathbb{K}$ shows that we can identify the symmetric space $\mathbb{G} / \mathbb{K}$ with $\overline{\mathbb{N}} \times \mathfrak{a}$ using the map $(\bar{n}, H) \rightarrow \bar{n}(\exp H) \cdot \mathbf{0}$. The change of measure is $d z=C e^{2 \rho(H)} d \bar{n} d H$ where $d \bar{n}$ is a Haar measure on $\overline{\mathbb{N}}$ and $\rho=$ $\frac{1}{2} \sum_{\alpha \in \Sigma^{+}} \operatorname{dim}\left(\mathfrak{g}_{\alpha}\right) \alpha$. For any $H \in \mathfrak{a}$ and $\bar{n} \in \overline{\mathbb{N}}$ let $\delta_{H}(\bar{n})=(\exp H) \bar{n}(\exp (-H))$.

It is well known that $\delta_{H}(\bar{n}) \in \overline{\mathbb{N}}$ and the map $\bar{n}_{1} \rightarrow \bar{n}_{2}=\delta_{H}\left(\bar{n}_{1}\right)$ is a dilation of $\overline{\mathbb{N}}$ with $d \bar{n}_{2}=e^{-2 \rho(H)} d \bar{n}_{1}$. Let $P(\bar{n})=e^{-2 \rho(H(\bar{n}))}$ be the Poisson kernel on $\overline{\mathbb{N}}$. Most of our analysis on the group $\overline{\mathbb{N}}$ will be based on the following proposition: 
Proposition 3. The maximal operator

$$
M_{\varepsilon} \phi(\bar{n})=\sup _{H \in \mathfrak{a}} \int_{\overline{\mathbb{N}}}\left|\phi\left(\bar{n}\left(\delta_{H}(\bar{m})\right)\right)\right|\left(P(\bar{m})+P\left(\bar{m}^{-1}\right)\right)^{(1+\varepsilon) / 2} d \bar{m},
$$

is bounded on $L^{r}(\overline{\mathbb{N}})$ for all $\varepsilon>0$ and $r>1$.

Proposition 3 is essentially proved in [5]. Proposition 5.1 in [5] guarantees the fact that the maximal operator

$$
M_{\varepsilon}^{\prime} \phi(\bar{n})=\sup _{H \in \mathfrak{a}_{\mathbb{Z}}} \int_{\overline{\mathbb{N}}} \phi\left(\bar{n}\left(\delta_{H}(\bar{m})\right)\right)(P(\bar{m}))^{(1+\varepsilon) / 2} d m,
$$

is bounded on $L^{r}(\overline{\mathbb{N}})$ for any $\varepsilon>0$ and $r>1$ where $\mathfrak{a}_{\mathbb{Z}}$ is the lattice of points $H \in \mathfrak{a}$ with the property that $\alpha(H) \in \mathbb{Z}$ for any simple positive root $\alpha$ (the notation in [5] differs from our notation in the sense that $P(\bar{n})=e^{-2 \rho\left(H\left(\bar{n}^{-1}\right)\right)}$ in $[5])$. One can repeat the argument in [5] to show that the factor $(P(\bar{m}))^{(1+\varepsilon) / 2}$ in the definition of the operator $M_{\varepsilon}^{\prime}$ can be replaced by $\left(P\left(\bar{m}^{-1}\right)\right)^{(1+\varepsilon) / 2}$ and the resulting maximal operator is also bounded on $L^{r}(\overline{\mathbb{N}})$ for any $r>1$. Finally, in order to be able to take the supremum over all $H \in \mathfrak{a}$ in (2.2) one only needs to notice that

$$
P(\bar{m}) \approx P\left(\delta_{H}(\bar{m})\right)
$$

if $H \in \mathfrak{a}$ has the property that $\alpha(H) \in[-1,1]$ for any $\alpha \in \Sigma_{0}^{+}$(the notation $u \approx v$ means that there exists an absolute constant $C$ depending only on the group $\mathbb{G}$ such that $\left.C^{-1} u \leq v \leq C u\right)$. To prove (2.3) notice that if $\bar{m}=k(\bar{m})(\exp H(\bar{m})) n(\bar{m})$ then $H\left(\delta_{H}(\bar{m})\right)=H(\bar{m})-H+H((\exp H) k(\bar{m}))$ so $\left|\rho\left(H\left(\delta_{H}(\bar{m})\right)-H(\bar{m})\right)\right| \leq C$. Along this line one can also prove that if $\mathcal{D}$ is a small, open, relatively compact set in $\overline{\mathbb{N}}$ then

$$
P(\bar{n}) \approx P(\bar{m} \cdot \bar{n})
$$

for all $\bar{m} \in \mathcal{D}$ and $\bar{n} \in \overline{\mathbb{N}}$.

\section{Proof of Theorem $1 b$}

It is more convenient to prove directly Theorem $1 \mathrm{~b}$ and obtain Theorem $1 \mathrm{a}$ as a consequence. We divide the proof of the theorem into four steps. First, we identify naturally the symmetric space $\mathbb{X}$ with $\overline{\mathbb{N}} \times \mathfrak{a}$ and describe the balls $B_{i}$ after this identification in (3.1). The basic idea of our approach is to associate to any ball $B_{i}=B\left(z_{i}, r_{i}\right) \in \mathcal{F}$ a certain subset $E_{i}=E\left(\bar{n}_{i}, H_{i}, r_{i}\right)$ defined in (3.2) (called the "end" of the ball) that carries a positive proportion of the volume of the ball $B_{i}$. In addition, the sets $E\left(\bar{n}_{i}, H_{i}, r_{i}\right)$ are product subsets of $\overline{\mathbb{N}} \times \mathfrak{a}$ and it turns out that the family of sets of the form $E(\bar{n}, H, r)$ has the covering property $V_{s}$ for all $s<\infty$. This enables us to select a suitable finite subset $J \subset I$ for which we prove that the two inequalities in (1.1) hold.

Step 1. Main construction: "ends" of balls. 
The Killing form $B$ on $\mathfrak{g}$ induces a positive definite scalar product on $\mathfrak{a}$ given by $\langle G, H\rangle=B(G, H)$. For any $H \in \mathfrak{a}$ let $|H|=\langle H, H\rangle^{1 / 2},|\rho|=\sup _{|H|=1} \rho(H)$ and $H_{\rho}$ the element of the sphere $|H|=1$ with the property that $|\rho|=\rho\left(H_{\rho}\right)$; one clearly has $\rho(H)=|\rho|\left\langle H, H_{\rho}\right\rangle$ for any $H \in \mathfrak{a}$. Let $P_{\rho}$ be the hyperplane (of dimension $\ell-1$ ) in $\mathfrak{a}$ defined by the equation $\rho\left(H^{\prime}\right)=0$ (i.e., the vector $H_{\rho}$ is perpendicular to the hyperplane $\left.P_{\rho}\right)$ and let $H=\left(x H_{\rho}, H^{\prime}\right), x \in \mathbb{R}, H^{\prime} \in P_{\rho}$ be the coordinates of $H$ in the natural identification $\mathfrak{a}=\mathbb{R} H_{\rho} \times P_{\rho}$. It is well known that $H_{\rho} \in \overline{\mathfrak{a}^{+}}$. We will assume from now on that $\ell$, the dimension of the Lie algebra $\mathfrak{a}$, is $\geq 2$ i.e., the hyperplane $P_{\rho}$ is not degenerate (only straightforward modifications are needed in the easier case $\ell=1$ ).

Notice that we can assume that all the balls in the statement of Theorem $1 \mathrm{~b}$ have large radius, say $\geq 2$. This is simply because small balls satisfy the usual doubling property $B(z, r) \approx B(z, 2 r)$ and thus the family of balls of radius $\leq 2$ has the simple covering property $V_{\infty}$. We can also assume that the set $I$ is finite. Let $\mathfrak{B}(r)=\{H \in \mathfrak{a}:|H|<r\}$ and we fix a small constant $c_{0}$ with the property that the set $\mathfrak{E}(r)=\left\{H=x H_{\rho}+H^{\prime}: x \in(r-3 / 2, r-1 / 2), H^{\prime} \in P_{\rho},\left|H^{\prime}\right|<\right.$ $\left.c_{0} r^{1 / 2}\right\}$ is included in $\mathfrak{B}(r-1 / 4)$ for any $r \geq 2$.

Using the map $(\bar{n}, H) \rightarrow \bar{n}(\exp H)$ we identify the symmetric space $\mathbb{X}$ with $\overline{\mathbb{N}} \times \mathfrak{a}$ and the relevant measure on $\overline{\mathbb{N}} \times \mathfrak{a}$ corresponding to this identification is $d \mu=e^{2 \rho(H)} d \bar{n} d H$. The letters $G, H$, possibly with subscripts and superscripts, will be used to denote various elements of $\mathfrak{a}$ and $\bar{m}, \bar{n}$ will denote elements of $\bar{N}$. For any $H \in \mathfrak{a}$ and $r>0$ let $\mathcal{D}(r, H)=\left\{\bar{n} \in \overline{\mathbb{N}}:[\bar{n}(\exp H)]^{+} \in \mathfrak{B}(r)\right\}$. By Konstant's convexity theorem, the set $\mathcal{D}(r, H)$ is non-empty if and only if $H \in \mathfrak{B}(r)$. If $\mathbf{0}=\{\mathbb{K}\}$ is the origin of the space $\mathbb{X}$ then $B(\mathbf{0}, r)=\left\{k\left(\exp H^{+}\right) \cdot \mathbf{0}\right.$ : $\left.k \in \mathbb{K}, H^{+} \in \mathfrak{B}(r) \cap \overline{\mathfrak{a}^{+}}\right\}=\{\bar{n}(\exp H) \cdot \mathbf{0}: H \in \mathfrak{B}(r), \bar{n} \in \mathcal{D}(r, H)\}$. For any $i \in I$ let $\left(\bar{n}_{i}, H_{i}\right)$ be the unique element of $\overline{\mathbb{N}} \times \mathfrak{a}$ with the property the $\bar{n}_{i}\left(\exp H_{i}\right) \cdot \mathbf{0}$ is the center of the ball $B_{i}$ and let $r_{i} \geq 2$ be the radius of the ball. The ball $B_{i}=B\left(\bar{n}_{i}\left(\exp H_{i}\right) \cdot \mathbf{0}, r_{i}\right)$ can be naturally identified with a set in $\overline{\mathbb{N}} \times \mathfrak{a}$ :

$$
\begin{aligned}
& B_{i} \equiv B\left(\bar{n}_{i}, H_{i}, r_{i}\right)= \\
& \quad\left\{\left(\bar{n}_{i} \delta_{H_{i}}(\bar{n}), H_{i}+H\right) \in \overline{\mathbb{N}} \times \mathfrak{a}: H \in \mathfrak{B}\left(r_{i}\right), \bar{n} \in \mathcal{D}\left(r_{i}, H\right)\right\} .
\end{aligned}
$$

Let $\mathcal{D} \subset \overline{\mathbb{N}}$ be a small, relatively compact open neighborhood of the origin of $\overline{\mathbb{N}}$ with the property that $\left|\bar{n}^{+}\right|<1 / 4$ for any $\bar{n} \in \mathcal{D}$. Since

$$
\begin{aligned}
\left|[\bar{n}(\exp H)]^{+}\right|=d(\bar{n}(\exp H) \cdot \mathbf{0}, \mathbf{0}) \leq \\
\quad d(\bar{n}(\exp H) \cdot \mathbf{0}, \bar{n} \cdot \mathbf{0})+d(\bar{n} \cdot \mathbf{0}, \mathbf{0})=|H|+\left|\bar{n}^{+}\right|
\end{aligned}
$$

it follows that for any $\bar{n} \in \mathcal{D}$ and $H \in \mathfrak{E}(r)$ one has $[\bar{n}(\exp H)]^{+} \in \mathfrak{B}(r)$. Therefore the set

$$
E_{i}=E\left(\bar{n}_{i}, H_{i}, r_{i}\right)=\left\{\left(\bar{n}_{i} \delta_{H_{i}}(\bar{n}), H_{i}+H\right) \in \overline{\mathbb{N}} \times \mathfrak{a}: H \in \mathfrak{E}\left(r_{i}\right), \bar{n} \in \mathcal{D}\right\}
$$


is a subset of $B\left(\bar{n}_{i}, H_{i}, r_{i}\right)$; in addition, since $e^{2 \rho(H)} \approx e^{2|\rho| r_{i}}$ if $H \in \mathfrak{E}\left(r_{i}\right)$ one has

$$
\mu\left(E\left(\bar{n}_{i}, H_{i}, r_{i}\right)\right) \approx e^{2|\rho| r_{i}} r_{i}^{(\ell-1) / 2} \approx\left|B_{i}\right|,
$$

(it is shown in [7] that the volume of a ball of radius $r \geq 2$ in $\mathbb{X}$ is proportional to $\left.e^{2|\rho| r} r^{(\ell-1) / 2}\right)$ therefore the set $E\left(\bar{n}_{i}, H_{i}, r_{i}\right)$ is product subset of $\overline{\mathbb{N}} \times \mathfrak{a}$ that captures a positive fraction of the volume of the ball $B\left(\bar{n}_{i}, H_{i}, r_{i}\right)$.

Step 2. Selection of the subset $J$.

We will now show the family $\mathcal{F}_{1}$ of subsets of $\overline{\mathbb{N}} \times \mathfrak{a}$ of the form $E(\bar{n}, H, r)=$ $\left\{\left(\bar{n} \delta_{H}(\bar{m}), H+G\right): G \in \mathfrak{E}(r), \bar{m} \in \mathcal{D}\right\}$ parametrized over $\bar{n} \in \overline{\mathbb{N}}, H \in \mathfrak{a}$ and $r \geq 2$ has the covering property $V_{s}$ for all $s<\infty$. The proof of this fact and the rest of the proof of the theorem will be based on working with a family of maximal operators on $(\overline{\mathbb{N}} \times \mathfrak{a}, d \mu)$. For any $\varepsilon \in(0,1]$ and any locally integrable function $f$ let

$$
M_{\varepsilon} f(\bar{n}, H)=\sup _{G \in \mathfrak{a}} \int_{\overline{\mathbb{N}}}\left|f\left(\bar{n}\left(\delta_{G}(\bar{m})\right), H\right)\right|\left(P(\bar{m})+P\left(\bar{m}^{-1}\right)\right)^{(1+\varepsilon) / 2} d \bar{m},
$$

and for any $\varepsilon, \delta \in(0,1]$ let

$$
\begin{aligned}
& A_{\varepsilon, \delta} f(\bar{n}, H)= \\
& \qquad \int_{y \leq 2}\left(\sup _{R>0} \int_{\left|G^{\prime}\right|<1, G^{\prime} \in P_{\rho}} M_{\varepsilon} f\left(\bar{n}, H+y H_{\rho}+R G^{\prime}\right) d G^{\prime}\right) e^{2|\rho| \delta y} d y .
\end{aligned}
$$

Lemma 4. The operator $A_{\varepsilon, \delta}$ is bounded on $L^{r}(\overline{\mathbb{N}} \times \mathfrak{a}, d \mu)$ if $r>1 / \delta$ :

$$
\left\|A_{\varepsilon, \delta} f\right\|_{L^{r}(\overline{\mathbb{N}} \times \mathfrak{a}, d \mu)} \leq C_{r, \varepsilon, \delta}\|f\|_{L^{r}(\overline{\mathbb{N}} \times \mathfrak{a}, d \mu)} \text { if } r>1 / \delta .
$$

Proof of Lemma 4. Notice that we can identify the measure space $(\overline{\mathbb{N}} \times \mathfrak{a}, d \mu)$ with $\left(\overline{\mathbb{N}} \times \mathbb{R} H_{\rho} \times P_{\rho}, e^{2|\rho| x} d \bar{n} d x d H^{\prime}\right)$ and the maximal operator $A_{\varepsilon, \delta}$ is the composition of the operator $M_{\varepsilon}$ acting on the $\bar{n}$ variable, the usual (Euclidean) maximal operator acting on $H^{\prime} \in P_{\rho}$ and the operator $T \phi(x)=\int_{y \leq 2} \phi(x+y) e^{2|\rho| \delta y} d y$. By Proposition 3, the first two maximal operators are bounded on $L^{r}\left(\overline{\mathbb{N}} \times \mathbb{R} H_{\rho} \times\right.$ $\left.P_{\rho}, e^{2|\rho| x} d \bar{n} d x d H^{\prime}\right)$ for any $r>1$; also, by Minkowski's inequality for integrals

$$
\begin{aligned}
\left(\int_{\mathbb{R}}|T \phi(x)|^{r} e^{2|\rho| x} d x\right)^{1 / r} & \leq \int_{(-\infty, 2]}\left(\int_{\mathbb{R}}|f(x+y)|^{r} e^{2|\rho| \delta y r} e^{2|\rho| x} d x\right)^{1 / r} d y \\
& \leq C_{\delta r}\|f\|_{L^{r}\left(\left(\mathbb{R} . e^{2|\rho| x} d x\right)\right)}
\end{aligned}
$$

if $\delta r>1$ and this completes the proof of the lemma.

By Proposition 2, in order to prove that the family of sets $\mathcal{F}_{1}$ has the covering property $V_{s}$ for all $s<\infty$ it suffices to show that the maximal operator

$$
\mathcal{M}_{\mathcal{F}_{1}}(\bar{n}, H)=\sup _{(\bar{n} . H) \in E \in \mathcal{F}_{1}} \frac{1}{\mu(E)} \int_{E} f(\bar{m}, G) d \mu(\bar{m}, G),
$$

is bounded on $L^{r}((\overline{\mathbb{N}} \times \mathfrak{a}, d \mu))$ for all $r>1$. If $E=E\left(\bar{n}^{0}, H^{0}, r^{0}\right)$ and $(\bar{n}, H) \in$ $E\left(\bar{n}^{0}, H^{0}, r^{0}\right)$ then $\bar{n}=\bar{n}^{0} \delta_{H^{0}}\left(\bar{m}^{0}\right)$ and $H=H^{0}+G^{0}$ for some $\bar{m}^{0} \in \mathcal{D}$ and 
$G^{0} \in \mathfrak{E}\left(r^{0}\right)$. Clearly $P(\bar{m}) \geq c$ if $\bar{m} \in \mathcal{D} \cdot \mathcal{D}$ and $e^{2 \rho(G)} \approx e^{2|\rho| r^{0}}$ if $G \in \mathfrak{E}\left(r^{0}\right)$. Therefore, using (3.3) and taking $R=2 c_{0}\left(r^{0}\right)^{1 / 2}$ in (3.5) and $G=H^{0}$ in (3.4) we have

$$
\begin{aligned}
& \frac{1}{\mu\left(E\left(\bar{n}^{0}, H^{0}, r^{0}\right)\right)} \int_{E\left(\bar{n}^{0}, H^{0}, r^{0}\right)} f(\bar{m}, G) e^{2 \rho(G)} d \bar{m} d G \\
& \leq C e^{-2|\rho| r^{0}}\left(r^{0}\right)^{-(\ell-1) / 2} \int_{\mathfrak{E}\left(r^{0}\right)} \int_{\mathcal{D}} f\left(\bar{n}^{0} \delta_{H^{0}}(\bar{m}), H^{0}+G\right) e^{2 \rho(G)} d m d G \\
& \leq C\left(r^{0}\right)^{-(\ell-1) / 2} \int_{\mathfrak{E}\left(r^{0}\right)} M_{1} f\left(\bar{n}, H+G-G^{0}\right) d G \leq C A_{1,1} f(\bar{n}, H) .
\end{aligned}
$$

By Lemma 4 the maximal operator $\mathcal{M}_{\mathcal{F}_{1}}$ is bounded on $L^{r}((\overline{\mathbb{N}} \times \mathfrak{a}, d \mu))$ for any $r>1$. By Proposition 2 and (2.1), one can select a subset $J \subset I$ such that for any $s<\infty$

$$
\left\|\sum_{j \in J} \chi_{E_{j}}\right\|_{L^{s}((\overline{\mathbb{N}} \times \mathfrak{a}, d \mu))} \leq C_{s} \mu\left(\cup_{i \in I} B_{i}\right)^{1 / s},
$$

and for any $i \in I$

$$
\mu\left(E_{i} \cap\left(\cup_{j \in J} E_{j}\right)\right) \geq \mu\left(E_{i}\right) / 2 .
$$

Step 3. Proof of (1.1)(ii).

We will now prove that the inequalities in (1.1) hold for the set $J$ selected above. We start with (1.1)(ii) and notice that it suffices to prove that if $1 \leq q<2$ then

$$
\left\|\sum_{j \in J} \chi_{B_{j}}\right\|_{L^{q}((\overline{\mathbb{N}} \times \mathfrak{a}, d \mu))} \leq C_{q} \mu\left(\cup_{i \in I} B_{i}\right)^{1 / q},
$$

where $B_{i}$ are the sets defined in (3.1). This will follow easily once we prove that for any ball $B_{j}=B\left(\bar{n}_{j}, H_{j}, r_{j}\right)$ and any $\varepsilon>0$ one has

$$
\int_{B\left(\bar{n}_{j}, H_{j}, r_{j}\right)} f d \mu \leq C_{\varepsilon} \int_{E\left(\bar{n}_{j}, H_{j}, r_{j}\right)} A_{\varepsilon, 1 / 2-\varepsilon} f d \mu
$$

for any locally integrable function $f$. In particular, it suffices to prove that for any point in $E\left(\bar{n}_{j}, H_{j}, r_{j}\right)$ i.e., of the form $\left(\bar{n}_{j} \delta_{H_{j}}\left(\bar{n}^{0}\right), H_{j}+x^{0} H_{\rho}+H^{0^{\prime}}\right)$ with $\bar{n}^{0} \in \mathcal{D}, x^{0} \in\left(r_{j}-3 / 2, r_{j}-1 / 2\right), H^{0^{\prime}} \in P_{\rho}$ and $\left|H^{0^{\prime}}\right|<c_{0} r_{j}{ }^{1 / 2}$ one has

$$
\begin{gathered}
\int_{B\left(\bar{n}_{j}, H_{j}, r_{j}\right)} f(\bar{n}, H) d \mu(\bar{n}, H) \leq \\
\quad C_{\varepsilon} e^{2|\rho| r_{j}} r_{j}{ }^{(\ell-1) / 2} A_{\varepsilon, \frac{1}{2}-\varepsilon} f\left(\bar{n}_{j} \delta_{H_{j}}\left(\bar{n}^{0}\right), H_{j}+x^{0} H_{\rho}+H^{0^{\prime}}\right) .
\end{gathered}
$$

To prove (3.10) observe first that if $\bar{n} \in \mathcal{D}\left(r_{j}, H\right)$ then

$$
P(\bar{n}) \geq e^{2\left(\rho(H)-|\rho| r_{j}\right)} .
$$


Indeed, if $\bar{n}(\exp H)=k_{1}\left(\exp H^{+}\right) k_{2}, k_{1}, k_{2} \in \mathbb{K}, H^{+} \in \mathfrak{B}\left(r_{j}\right)$ then $H(\bar{n})+H=$ $H\left[\left(\exp H^{+}\right) k_{2}\right]$ and (3.11) follows from Konstant's convexity theorem. It follows from (3.11) and (2.4) that

$$
\int_{\mathcal{D}\left(r_{j}, H\right)} f\left(\bar{n}_{j} \delta_{H_{j}}(\bar{n}), H_{j}+H\right) d \bar{n} \leq C M_{\varepsilon} f\left(\bar{n}_{j} \delta_{H_{j}}\left(\bar{n}^{0}\right), H_{j}+H\right) e^{(1+\varepsilon)\left(|\rho| r_{j}-\rho(H)\right)}
$$

therefore

$$
\begin{aligned}
& \int_{B\left(\bar{n}_{j}, H_{j}, r_{j}\right)} f(\bar{n}, H) d \mu(\bar{n}, H) \leq \\
& C \int_{\mathfrak{B}\left(r_{j}\right)} M_{\varepsilon} f\left(\bar{n}_{j} \delta_{H_{j}}\left(\bar{n}^{0}\right), H_{j}+H\right) e^{(1+\varepsilon)|\rho| r_{j}+(1-\varepsilon) \rho(H)} d H .
\end{aligned}
$$

Let $H=\left(\left(x^{0}+y\right) H_{\rho}\right)+\left(H^{0^{\prime}}+H^{\prime}\right)$ where $y \in \mathbb{R}, H^{\prime} \in P_{\rho}$ have the property that $\left(x^{0}+y\right)^{2}+\left|H^{0^{\prime}}+H^{\prime}\right|^{2} \leq r_{j}^{2}$. Notice that this region is included in the region $y \leq 2,\left|H^{\prime}\right| \leq C r_{j}{ }^{1 / 2}(1+|y|)^{1 / 2}$. If one lets $R=C r_{j}{ }^{1 / 2}(1+|y|)^{1 / 2}$ in (3.5) and notices that $e^{(1-\varepsilon)|\rho| y}(1+|y|)^{(\ell-1) / 2} \leq C_{\varepsilon} e^{(1-2 \varepsilon)|\rho| y}$ if $y \leq 2$, (3.10) follows and (3.9) follows from (3.3) and (3.10).

Let $p$ be such that $1 / p+1 / q=1$. Using (3.6) and (3.9) one has

$$
\begin{aligned}
\left\|\sum_{j \in J} \chi_{B_{j}}\right\| \|_{L^{q}((\overline{\mathbb{N}} \times \mathfrak{a}, d \mu))} & =\sup _{\|f\|_{p}=1} \int_{\overline{\mathbb{N}} \times \mathfrak{a}} f\left(\sum_{j \in J} \chi_{B_{j}}\right) d \mu \\
& \leq C_{\varepsilon} \sup _{\|f\|_{p}=1} \int_{\overline{\mathbb{N}} \times \mathfrak{a}} A_{\varepsilon, 1 / 2-\varepsilon} f\left(\sum_{j \in J} \chi_{E_{j}}\right) d \mu \\
& \leq C_{\varepsilon, q} \sup _{\|f\|_{p}=1}\left\|A_{\varepsilon, 1 / 2-\varepsilon} f\right\|_{p} \mu\left(\cup_{i \in I} B_{i}\right)^{1 / q} .
\end{aligned}
$$

Clearly (3.8) now follows from Lemma 4 if one chooses $\varepsilon$ such that $p(1 / 2-\varepsilon)>1$ which is equivalent to $\varepsilon<1 / q-1 / 2$.

Step 4. Proof of (1.1)(i).

Notice that it suffices to prove that

$$
\mu\left(\cup_{i \in I} B_{i}\right) \leq C \mu\left(\cup_{j \in J} E_{j}\right)
$$

where $B_{i}$, respectively $E_{i}$, are the sets defined in (3.1), respectively (3.2). For each $k \in\{0,1, \ldots\}$ let

$$
\begin{array}{r}
B_{i}^{k}=\left\{\left(\bar{n}_{i} \delta_{H_{i}}(\bar{n}), H_{i}+H\right) \in \overline{\mathbb{N}} \times \mathfrak{a}: H=x H_{\rho}+H^{\prime}, x \in\left[r_{i}-k-1, r_{i}-k\right],\right. \\
\left.H^{\prime} \in P_{\rho},\left|H^{\prime}\right| \leq 2 r_{i}^{1 / 2}(k+1)^{1 / 2}, P(\bar{n}) \geq e^{-2|\rho|(k+1)}\right\} .
\end{array}
$$


Using (3.11) and the definition (3.1) of the balls $B_{i}$, it follows easily that $B_{i} \subset \bigcup_{k=0}^{\infty} B_{i}^{k}$. Let $U^{0}=\cup_{j \in J} E_{j}$ and for any positive integer $k$ let $U^{k}=U^{0}-k H_{\rho}=$ $\left\{(\bar{n}, H):\left(\bar{n}, H+k H_{\rho}\right) \in U^{0}\right\}$. Let $f_{k}$ be the characteristic function of the set $U^{k}$. Using (3.7) we will prove that for any point $\left(\bar{n}^{0}, H^{0}\right) \in \underset{i \in I}{\cup} B_{i}^{k}$

$$
A_{\varepsilon, 1} f_{k}\left(\bar{n}^{0}, H^{0}\right) \geq c e^{-(1+\varepsilon)|\rho| k}(k+1)^{-(\ell-1) / 2} .
$$

Assuming this for a moment, it follows from Lemma 4 that for any $r>1$

$$
\begin{aligned}
\mu\left(\cup_{i \in I} B_{i}^{k}\right) \leq C_{r} \mu\left(U^{k}\right) e^{r(1+\varepsilon)|\rho| k}(k+1)^{r(\ell-1) / 2} & = \\
& C_{r} \mu\left(U^{0}\right) e^{(r(1+\varepsilon)-2)|\rho| k}(k+1)^{r(\ell-1) / 2},
\end{aligned}
$$

therefore, if one chooses $r>1$ and $\varepsilon>0$ with the property that $r(1+\varepsilon)<2$

$$
\mu\left(\cup_{i \in I} B_{i}\right) \leq \sum_{k=0}^{\infty} \mu\left(\cup_{i \in I} B_{i}^{k}\right) \leq C \mu\left(U^{0}\right),
$$

which proves (3.12).

It remains therefore to prove $(3.13)$. The point $\left(\bar{n}^{0}, H^{0}\right)$ belongs to the set $B_{i}^{k}$ for some $i \in I$ therefore $\bar{n}^{0}=\bar{n}_{i} \delta_{H_{i}}\left(\bar{m}^{0}\right), H^{0}=H_{i}+y^{0} H_{\rho}+G^{0^{\prime}}$ where

$$
\left\{\begin{array}{l}
P\left(\bar{m}^{0}\right) \geq e^{-2|\rho|(k+1)} ; \\
y^{0} \in\left[r_{i}-k-1, r_{i}-k\right] ; \\
\left|G^{0^{\prime}}\right| \leq 2 r_{i}^{1 / 2}(k+1)^{1 / 2}, G^{0^{\prime}} \in P_{\rho} .
\end{array}\right.
$$

Using the first inequality in (3.14) and (2.4) one has for any $H \in \mathfrak{a}$

$$
\begin{aligned}
M_{\varepsilon} f_{k}\left(\bar{n}^{0}, H\right) & \geq \int_{\overline{\mathbb{N}}} f_{k}\left(\bar{n}_{i} \delta_{H_{i}}\left(\bar{m}^{0} \bar{m}\right), H\right) P\left(\bar{m}^{-1}\right)^{(1+\varepsilon) / 2} d \bar{m} \\
& \geq \int_{\mathcal{D}} f_{k}\left(\bar{n}_{i} \delta_{H_{i}}(\bar{n}), H\right) P\left(\bar{n}^{-1} \bar{m}^{0}\right) d \bar{n} \\
& \geq c e^{-(1+\varepsilon)|\rho| k} \int_{\mathcal{D}} f_{k}\left(\bar{n}_{i} \delta_{H_{i}}(\bar{n}), H\right) d \bar{n}
\end{aligned}
$$

If one takes $R=3 r_{i}^{1 / 2}(k+1)^{1 / 2}$ and restricts $y$ to $[-2,2]$ in (3.5) it follows that

$$
\begin{aligned}
& A_{\varepsilon, 1} f_{k}\left(\bar{n}^{0}, H^{0}\right) \\
& \geq c e^{-(1+\varepsilon)|\rho| k}(k+1)^{-(\ell-1) / 2} r_{i}^{-(\ell-1) / 2} \\
& \quad \int_{r_{i}-3 / 2}^{r_{i}-1 / 2} \int_{\left|H^{\prime}\right| \leq c_{0} r_{i}^{1 / 2}} \int_{\mathcal{D}} f_{k}\left(\bar{n}_{i} \delta_{H_{i}}(\bar{n}), H_{i}+(x-k) H_{\rho}+H^{\prime}\right) d \bar{n} d H^{\prime} d x \\
& \geq c e^{-(1+\varepsilon)|\rho| k}(k+1)^{-(\ell-1) / 2} r_{i}^{-(\ell-1) / 2} e^{2|\rho|\left(k-r_{i}\right)} \int_{E_{i}-k H_{\rho}} f_{k}(\bar{m}, G) d \mu(\bar{m}, G) .
\end{aligned}
$$

It follows from (3.7) that

$$
\int_{E_{i}-k H_{\rho}} f_{k}(\bar{m}, G) d \mu(\bar{m}, G) \geq \mu\left(E_{i}-k H_{\rho}\right) / 2 \approx r_{i}^{(\ell-1) / 2} e^{2|\rho|\left(r_{i}-k\right)},
$$


and (3.13) follows from the last two inequalities.

\section{Sharpness of the Theorems}

We will now prove that the maximal operator $\mathcal{M}_{\mathcal{F}}$ is not bounded from the Lorentz space $L^{2, \alpha}(\mathbb{X})$ to $L^{2, \infty}(\mathbb{X})$ if $\alpha>1$. The definition and some simple properties of Lorentz spaces may be found, for example, in [6, Chapter V]. It is natural to look for counterexamples $g_{\beta}: \mathbb{X} \rightarrow \mathbb{R}_{+}$of the form

$$
g_{\beta}(z)=e^{-|\rho| d(\mathbf{0}, z)}(1+d(\mathbf{0}, z))^{-\beta},
$$

for certain suitable exponents $\beta$. Since

$$
|B(\mathbf{0}, N+1) \backslash B(\mathbf{0}, N)| \approx e^{2|\rho| N}(N+1)^{(\ell-1) / 2},
$$

if $N \geq 0$, it follows that the nonincreasing rearrangement $g_{\beta}{ }^{*}:(0, \infty) \rightarrow \mathbb{R}_{+}$of the function $g_{\beta}$ has the property that

$$
\left\{\begin{array}{l}
g_{\beta}{ }^{*}(t) \approx 1 \text { if } t \in(0,1] ; \\
g_{\beta}{ }^{*}\left(e^{2|\rho| x}(x+1)^{(\ell-1) / 2}\right) \approx e^{-|\rho| x}(x+1)^{-\beta} \text { if } x \geq 0 .
\end{array}\right.
$$

Therefore $g_{\beta} \in L^{2, \alpha}(\mathbb{X})$ if

$$
\beta>\frac{\ell-1}{4}+\frac{1}{\alpha} .
$$

We will now show that if $N \geq 1$ is a large integer, $k \in \mathbb{K}$ and $H \in \mathfrak{E}(N)$ (same notation as in the previous section) then

$$
\mathcal{M}_{\mathcal{F}} g_{\beta}(k(\exp H) \cdot \mathbf{0}) \geq c e^{-|\rho| N} N^{-\beta+1} .
$$

Since the functions $g_{\beta}$ are $\mathbb{K}$-invariant, it suffices to prove (4.2) for $k=e$-the identity element of the group $\mathbb{G}$. Notice that $(\exp H) \cdot \mathbf{0} \in B\left(\left(\exp \left(N H_{\rho} / 2\right)\right)\right.$. $\mathbf{0}, N / 2+2)$ if $H \in \mathfrak{E}(N)$ therefore

$$
\mathcal{M}_{\mathcal{F}} g_{\beta}((\exp H) \cdot \mathbf{0}) \geq \frac{1}{\left|B_{N}\right|} \int_{B_{N}} g_{\beta}\left(z^{\prime}\right) d z^{\prime},
$$

where $B_{N}=B\left(\left(\exp \left(N H_{\rho} / 2\right)\right) \cdot \mathbf{0}, N / 2+2\right)$. Let

$$
\mathfrak{R}(N)=\left\{G=y H_{\rho}+G^{\prime} \in \mathfrak{a}: y \in[N / 3,2 N / 3], G^{\prime} \in P_{\rho},\left|G^{\prime}\right| \leq c_{1} N^{1 / 2}\right\},
$$

where $c_{1}$ is a small constant. If $G \in \mathfrak{R}(N)$ and $\bar{m} \in \delta_{G / 2}(\mathcal{D})$ then

$$
\begin{aligned}
& d\left(\bar{m}(\exp G) \cdot \mathbf{0},\left(\exp \left(N H_{\rho} / 2\right)\right) \cdot \mathbf{0}\right) \\
& \quad \leq|G| / 2+d\left(\bar{m}(\exp (G / 2)) \cdot \mathbf{0},\left(\exp \left(N H_{\rho} / 2\right)\right) \cdot \mathbf{0}\right) \\
& \quad \leq|G| / 2+\left|N H_{\rho}-G\right| / 2+d((\exp (-G / 2)) \bar{m}(\exp (G / 2)) \cdot \mathbf{0}, \mathbf{0})<N / 2+2 .
\end{aligned}
$$


The last of the inequalities in the sequence above holds if $c_{1}$ is small enough.

Thus

$$
\begin{aligned}
& \frac{1}{\left|B_{N}\right|} \int_{B_{N}} g_{\beta}\left(z^{\prime}\right) d z^{\prime} \geq \\
& \quad c e^{-|\rho| N} N^{-(\ell-1) / 2} \int_{\mathfrak{R}(N)} \int_{\delta_{G / 2}(\mathcal{D})} g_{\beta}(\bar{m}(\exp G) \cdot \mathbf{0}) d \bar{m} e^{2 \rho(G)} d G .
\end{aligned}
$$

On the other hand if $\bar{m}=\delta_{G / 2}(\bar{n}), \bar{n} \in \mathcal{D}$ then

$$
d(\bar{m}(\exp G) \cdot \mathbf{0}, \mathbf{0})=d((\exp (G / 2)) \bar{n}(\exp (G / 2)) \cdot \mathbf{0}, \mathbf{0}) \leq|G|+1 / 4
$$

therefore

$$
g_{\beta}(\bar{m}(\exp G) \cdot \mathbf{0}) \geq c e^{-|\rho| G}|G|^{-\beta} .
$$

One clearly has $\int_{\delta_{G / 2}(\mathcal{D})} 1 d \bar{m} \approx e^{-\rho(G)}$ and $\int_{\mathfrak{R}(N)} 1 d G \approx N^{(\ell-1) / 2+1}$. The main estimate (4.2) now follows from (4.3), (4.4), (4.5) and the observation that $e^{|\rho||G|} \approx e^{\rho(G)}$ if $G \in \mathfrak{R}(N)$. Since the volume of the set of points in $\mathbb{X}$ of the form $k(\exp H) \cdot \mathbf{0}, k \in \mathbb{K}, H \in \mathfrak{E}(N)$ is proportional to $e^{2|\rho| N} N^{(\ell-1) / 2}$ it follows from (4.2) that

$$
\left\|\mathcal{M}_{\mathcal{F}} g_{\beta}\right\|_{L^{2, \infty}(\mathbb{X})} \geq c N^{(\ell-1) / 4+1-\beta} .
$$

Therefore $\mathcal{M}_{\mathcal{F}} g_{\beta} \notin L^{2, \infty}(\mathbb{X})$ if $\beta<(\ell-1) / 4+1$ which is compatible with (4.1) if $\alpha>1$.

\section{References}

[1] J.L. Clerc and E.M. Stein, $L^{p}$-multipliers for noncompact symmetric spaces, Proc. Natl. Acad. Sci. U.S.A. 71 (1974), 3911-3912.

[2] A. Cordoba and R. Fefferman, A geometric proof of the strong maximal theorem, Ann. Math. 102 (1975), 95-100.

[3] S. Helgason, Geometric analysis on symmetric spaces, Mathematical Surveys and Monographs, 39, American Mathematical Society, Providence, RI, 1994.

[4] A.D. Ionescu, An endpoint estimate for the Kunze-Stein phenomenon and related maximal operators, preprint 1999.

[5] P. Sjögren, Admissible convergence of Poisson integrals in symmetric space, Ann. Math. 124 (1986), 313-335.

[6] E.M. Stein and G. Weiss, Introduction to Fourier analysis on Euclidean spaces, Princeton Mathematical Series, No. 32, Princeton University Press, Princeton, N.J., 1971.

[7] J.O.Strömberg, Weak type $L^{1}$ estimates for maximal functions on noncompact symmetric spaces, Ann. Math. 114 (1981), 115-126.

Department of Mathematics, Princeton University, Princeton, NJ 08544

E-mail address: aionescu@math.princeton.edu 